due to the increasing importance of peripheral alpha-adrenoceptor stimulation once a maximal central effect has been reached. Differences in the sensitivities of central and peripheral alpha-adrenoceptors to clonidine have been reported. ${ }^{11-13}$

Thus the balance of the evidence favours peripheral stimulation as the cause of drug resistance in patients on very high doses of clonidine. Our observations suggest that the use of high doses of clonidine may be associated with diminishing and not increasing hypotensive effects, and this should be borne in mind when the dose is being adjusted.

We thank Miss $P$ Tippett, Miss E Neill, and Mrs J Daniel for technical help and Miss A Davis and Miss B Edinborough, who kindly typed the manuscript.

LMHW is a fellow in clinical sciences of the National Health and Medical Research Council of Australia and is also supported by the Wellcome Trust. JLR is supported by the Wellcome Trust as a senior fellow in clinical science.

\section{References}

${ }^{1}$ Conolly, M E, in Central Action of Drugs in Blood Pressure Regulation, ed D S Davies and J L Reid, p 268. Kent, Pitman Medical, 1975.

${ }^{2}$ Macdougall, A I, et al, British Medical fournal, 1970, 3, 440.

3 Onesti, G, et al, Circulation Research, 1971, 28 and 29, suppl 11, p 11. Conolly, M E, et al, European fournal of Clinical Pharmacology, 1972, 4, 222.

5 Davies, D S, et al, Clinical Pharmacology and Therapeutics, in press.

6 Wing, L M H, et al, European fournal of Clinical Investigation, 1976, 6, 314. C

' Dollery, C T, et al, Clinical Pharmacology and Therapeutics, 1976, 19, 11.

Martin, L E, et al, British fournal of Pharmacology, 1976, 35, 695.

Hunyor, S N, et al, British Medical fournal, 1975, 4, 23.

${ }^{10}$ Kobinger, W, in Central Action of Drugs in Blood Pressure Regulation, ed D S Davies and J L Reid, p 181. Kent, Pitman Medical, 1975.

11 Schmitt, H, Schmitt, M, European fournal of Pharmacology, 1970, 9, 7. 12 Schmitt, H, Schmitt, M, and Fenard, S, European fournal of Pharmacology, 1971, 14, 98.

${ }^{13}$ Struyker, B H, et al, Life Sciences, 1974, 15, 887.

\title{
Thyrotoxicosis: relations between clinical state and biochemical changes during carbimazole treatment
}

\author{
C H MORTIMER, D C ANDERSON， P LIENDO-CH， R FISHER，V CHAN， M SELF， G M BESSER
}

British Medical fournal, 1977, 1, 138-141

\begin{abstract}
Summary
The relation between clinical and biochemical changes in thyrotoxicosis were studied in 12 patients with Graves's disease who were being treated with carbimazole. Clinical assessment (using the Crooks-Wayne index) was combined with the measurement of free thyroxine and triiodothyronine indices (FT4I and FT3I) and the assessment of two tissue markers of thyroid hormone actionsex-hormone-binding globulin (SHBG) levels and the thyrotrophin responses to TRH. In general the FT4I and FT3I fell rapidly once treatment was started, and returned to normal in one to four weeks, followed shortly by SHBG levels. The thyrotrophin response returned at this time in two patients, who still had borderline high levels of FT3I and SHBG. The clinical score fell more slowly and variably and was less closely related to any of the biochemical indices than these were to each other.

During the early phase of treatment with antithyroid drugs the clinical evaluation may be an unreliable indicator of persisting thyroid hormone excess, and when the patient seems clinically but not biochemically thyrotoxic the symptoms should be treated on their own merits with beta-blocking drugs and not with increased doses of antithyroid drugs.
\end{abstract}

Department of Endocrinology, Medical Professorial Unit, and Department of Chemical Pathology, St Bartholomew's Hospital, London EC1A 7BE

C H MORTIMER, MB, MRCP, research lecturer in medicine

D C ANDERSON, MD, MRCP, lecturer in medicine (now senior lecturer in medicine, Manchester Royal Infirmary)

P LIENDO-CH, MB, research fellow

R FISHER, MSC, biochemist

V CHAN, PHD, biochemist (now lecturer, Department of Medicine, Queen Mary Hospital, University of Hong Kong)

M SELF, FIMLT, technician

G M BESSER, MD, FRCP, professor of endocrinology

\section{Introduction}

Despite advances in the treatment of thyrotoxicosis after the introduction of antithyroid drugs some practical problems remain, particularly in gauging the doses required. Antithyroid drugs are directed at reducing thyroid hormone production, which is ultimately responsible for the symptoms and signs of thyrotoxicosis. Many of these clinical features result from secondary changes, however, including increased tissue sensitivity to catecholamines, which may not be rapidly reversed when the thyroid hormone excess is controlled. We examined the relation between clinical evidence of thyrotoxicosis, serum levels of thyroid hormones, and two objective biochemical markers of tissue response to thyroid hormones before and during carbimazole treatment. Our aim was to clarify the relation during treatment between circulating thyroid hormone levels, other objective biochemical changes, and the clinical manifestations of thyrotoxicosis.

\section{Patients and methods}

Five men and seven women aged 25 to 62 years with diffuse goitre and thyrotoxic Graves's disease were studied. After baseline observations were made carbimazole $45 \mathrm{mg} /$ day was given until clinical improvement was seen. Then the dose was reduced gradually on clinical grounds to $5-15 \mathrm{mg}$ /day for at least one year in all but three patients. One of these (case 1) developed a cutaneous rash during the seventh week, necessitating prednisolone treatment. The rash improved, but five weeks later leucopenia developed and treatment was changed to propylthiouracil and potassium iodide, followed by partial thyroidectomy. Another patient (case 2) elected to have a thyroidectomy at four months. One patient (case 3) arbitrarily varied her own dosage over the first 16 weeks so she did not follow a taperingdose regimen. Ten patients were also studied during the period immediately after stopping carbimazole; two of them (cases 4 and 5) suffered a recurrence of thyrotoxicosis.

Clinical status was assessed serially by a single observer with no knowledge of the biochemical changes. It was scored according to the Crooks-Wayne index. ${ }^{1}$ Scores of 6-31 suggest thyrotoxicosis. When the score was 5 or less clinical status was also assessed by the hypothyroid index of Billewicz et al. ${ }^{2}$ Results of this are not presented since no patient became clinically hypothyroid. 
Indices of free triiodothyronine (FT3I) and thyroxine (FT4I) were measured serially. One of the "tissue markers" of thyroid hormone action studied was the pituitary thyrotrophin response to thyrotrophinreleasing hormone (TRH), which is characteristically suppressed in untreated thyrotoxicosis by the thyroid hormones' action on the pituitary cells. The other was the plasma level of sex-hormonebinding globulin (SHBG), which is considerably raised in thyrotoxic patients of both sexes. ${ }^{3-5}$ This rise in SHBG may be a direct effect of thyroid hormones on hepatic SHBG synthesis or mediated in part at least by increased conversion of androgens to oestrogens. ${ }^{6}$ 7

Plasma SHBG binding-site concentration was measured as described. ${ }^{8}$ Serum protein-bound iodine (PBI) was measured by Technicon AutoAnalyzer Method $56^{\circ}$ and total serum T4 by competitive protein-binding (Thyopac-4). The thyroid hormone uptake test (THUT) was performed by measuring Sephadex uptake of ${ }^{125}$ I-labelled T3 (Thyopac-3). The FT4I was calculated from the PBI or T4 and the THUT result. ${ }^{10}$ Total serum T3 was measured by radioimmunoassay. ${ }^{11}$ The FT3I was calculated by substituting T3 and THUT values in the same formula as that used for the FT4I. Serum immunoreactive thyrotrophin was measured by double antibody radioimmunoassay ${ }^{12}$ and the results expressed as $\mathrm{mU} / \mathrm{l}$ of MRC standard 68/38 human thyrotrophin. The TRH tests were performed as described. ${ }^{13}$ Results were classified as either absent (thyrotrophin increment $<1.0 \mathrm{mU} / \mathrm{l}$ ), normal (values at 20 minutes 3.5-20.5 mU/1), impaired, or exaggerated. Assessment was carried out weekly for three to 12 weeks, then every two to four weeks until carbimazole treatment stopped, and then again at weekly intervals.

\section{Results}

\section{BASAL FINDINGS}

According to their clinical scores (18-31) all patients were clinically thyrotoxic before treatment. Formal scoring was not conducted at this stage in case 6 , but this patient was obviously thyrotoxic; his clinical score was raised two weeks later when he was first formally scored. FT4I levels were raised (119-330) in all but two patients (cases 5 and 7), and FT3I levels were raised in 11 patients (3-14.2) including both cases 5 and 7 . These patients were therefore considered to have T3 thyrotoxicosis. One patient (case 8) had a normal FT3I level but raised FT4I. Basal serum immunoreactive thyrotrophin was undetectable in all subjects except one (case 9), and in no case was there any rise after TRH. SHBG levels measured basally in all except case 6 were consistently raised, to a mean of $239 \mathrm{nmol} / 1$ (about $12.4 \mathrm{mg} / \mathrm{l}$ ) for the women and $208 \mathrm{nmol} / 1$ (about $10.8 \mathrm{mg} / \mathrm{l}$ ) for the men. These values were, respectively, 2.9 and 6.3 times the normal values. There was one exceptionally high basal level of $524 \mathrm{nmol} / \mathrm{l}$ (about $27.3 \mathrm{mg} / \mathrm{l}$ ) in case 1 , and when this value was excluded the mean value for the men was $3 \cdot 1$ times the normal value.

\section{RESPONSE TO TREATMENT}

The typical pattern of response is shown in fig 1 for case 10 during the first 16 weeks of treatment. Clinical improvement with a fall in the clinical score of over 3 was seen within the first one to three weeks in eight of the 12 patients and in the remainder after four to five weeks had elapsed. The clinical score became normal in three to 18 weeks in the group as a whole.

Of the results of biochemical measurements, the FT4I and FT3I started to return towards normal within the first one to two weeks of treatment. FT4I came into the normal range in one to four weeks. The FT3I fell to normal over the same period, but in five of the patients it rose again into the thyrotoxic range more than once over the next year. In four of these patients the FT3I remained raised despite the presence of clinical euthyroidism for three to 11 weeks. The pattern of changes in circulating total T3 and T4 levels did not differ from those seen in FT3I and FT4I. SHBG also fell significantly within the first week in four patients, and was lower in all patients after three weeks' treatment, becoming normal after one to nine weeks, except in two patients in whom it remained high for 22 and 35 weeks.

The rates of improvement of the different measurements seemed to be consistent, with FT3I and FT4I falling in parallel and rapidly followed by the SHBG, with the clinical score responding more slowly. In nine of the subjects the TRH response returned within two to four weeks of the FT3I and FT4I becoming normal. In two patients (cases 3 and 4 ) the thyrotrophin response to TRH did not return for a further 19 and 29 weeks. In each case the FT3I or SHBG, or both, remained borderline high, but the patients were clinically euthyroid.

One patient (case 3) showed an atypical response. She stopped her carbimazole for one week after seven weeks of treatment after an emotional upset. Initially she had shown a rapid improvement in all clinical and biochemical indices. After she stopped her carbimazole the FT3I and FT4I rose, and there was no further clinical improvement despite apparent resumption of treatment. The clinical score,

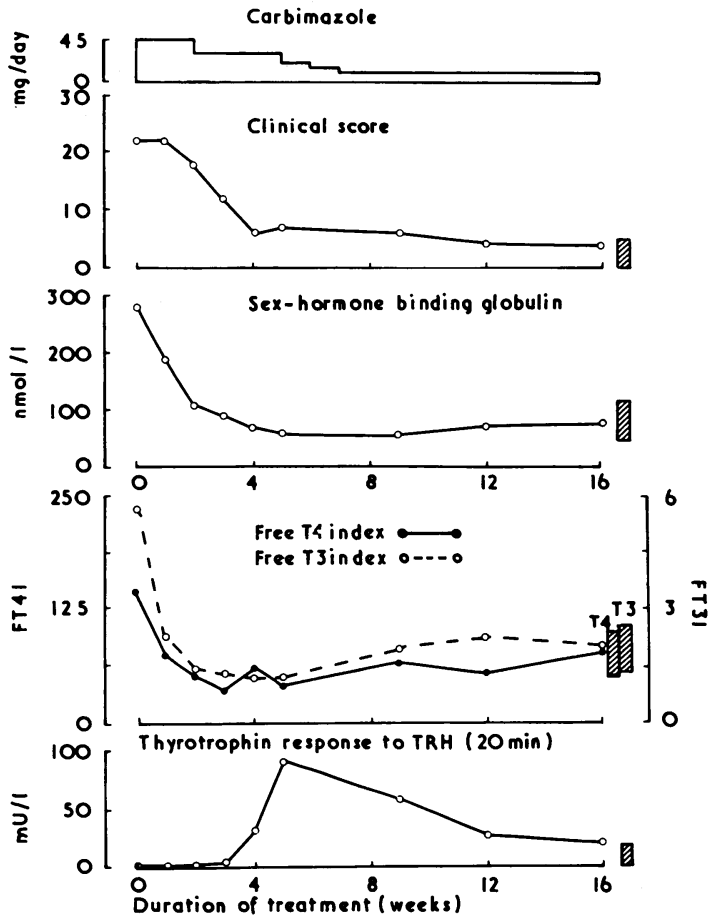

FIG 1-Case 10: 27-year-old woman. Typical response to carbimazole treatment of clinical score and biochemical markers of thyrotoxicosis. Hatched areas depict normal range.

Conversion: SI to traditional units-SHBG: $1 \mathrm{nmol} / 1 \approx$ $0.052 \mathrm{mg} / \mathrm{l}$.

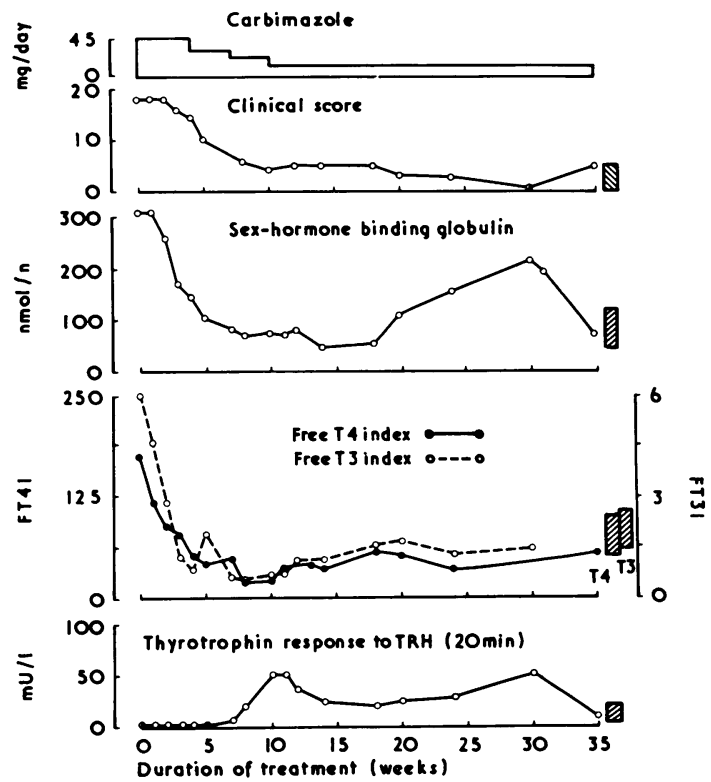

FIG 2-Case 11 : 59-year-old woman. Response to carbimazole treatment in patient with clinical euthyroidism despite low FT3I, FT4I, and exaggerated thyrotrophin response to TRH. Unexplained secondary SHBG rise occurred between 20 and 31 weeks. Hatched areas depict normal range. 
FT3I, and SHBG remained consistently at the upper limit of normal and the TRH response did not return.

There seemed to be a close relation between SHBG and the free thyroid hormone indices in the initial phase of treatment (see below), and subsequently in all but one woman (case 11). Her response is shown in fig 2. As in the other patients, there was an initial rapid fall in FT3I and FT4I with slower falls in SHBG and the clinical score. After eight weeks the low FT3I and FT4I and the exaggerated thyrotrophin response to TRH suggested biochemical hypothyroidism, although the patient remained clinically euthyroid. From 20-31 weeks there was a considerable rise in plasma SHBG, which could not be explained on the basis of changes in thyroid function. Oestrogens also stimulate SHBG production but the patient was not receiving oestrogen treatment, and the increase remains unexplained.
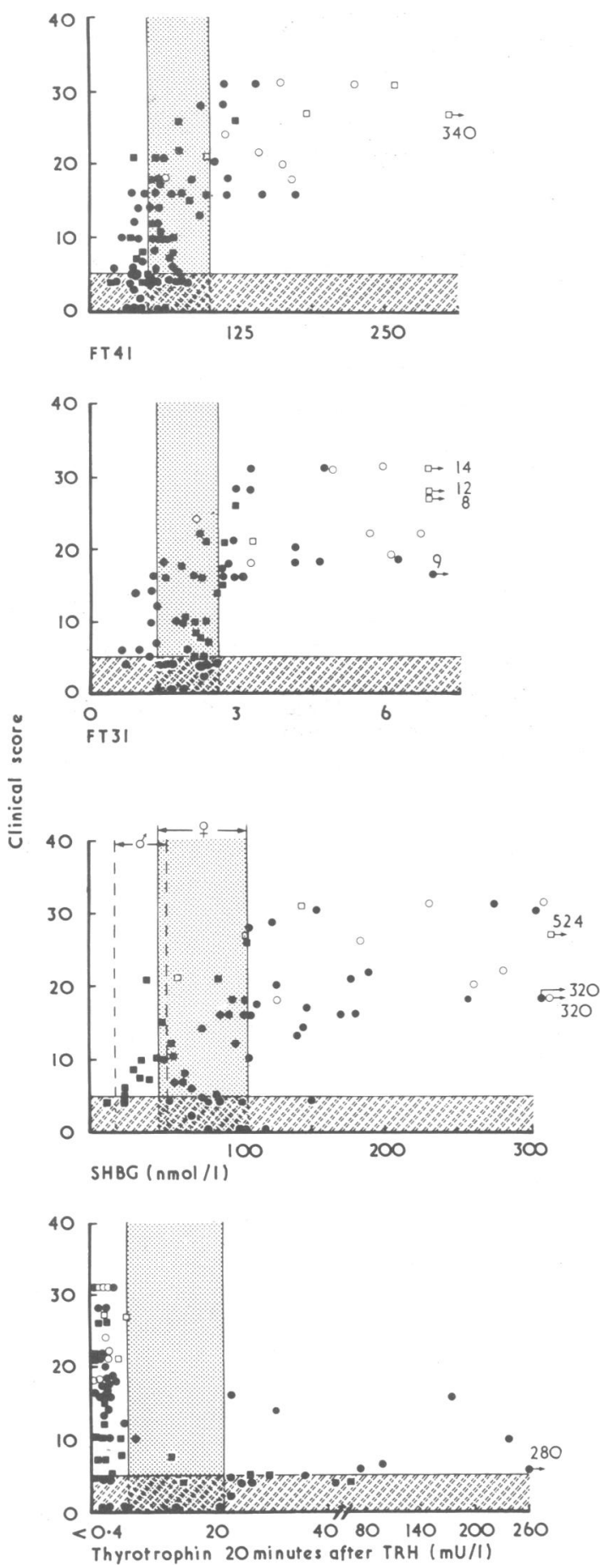

FIG 3-Relation between clinical score and FT4I, FT3I, SHBG, and the thyrotrophin response to TRH during first 12 weeks of carbimazole treatment. $(\triangle=$ Men and $\mathrm{O}=$ women before treatment. $\Delta=$ Men and $O=$ women during treatment). Hatched areas depict normal range.
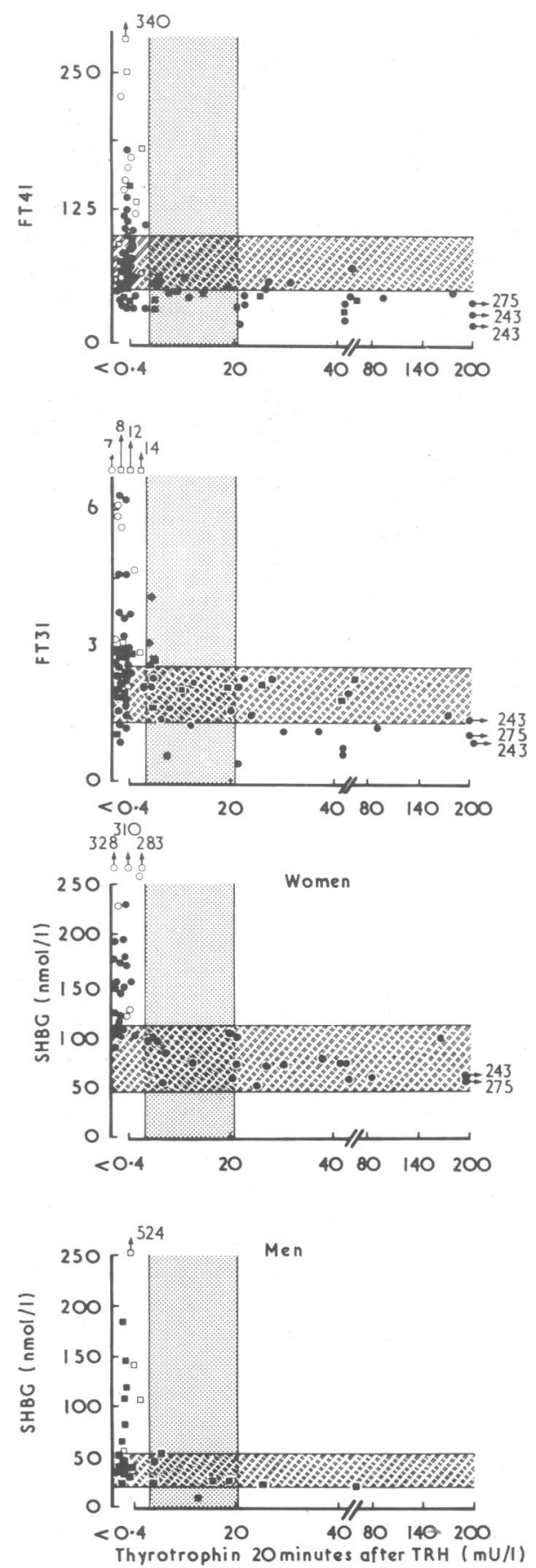

FIG 4-Relation between FT4I, FT3I, SHBG, and thyrotrophin response to TRH during first 12 weeks of carbimazole therapy $(\triangle=$ Men and $O=$ women before treatment. $\Delta=$ Men and $\mathbf{O}=$ women during treatment. Hatched areas depict normal range.

The sex difference in SHBG levels evident in normal subjects was also seen in thyrotoxicosis: the women had higher levels than men for a given degree of biochemical or clinical hyperthyroidism at all stages of treatment.

RELATION BETWEEN INDICES OF THYROID STATUS DURING TREATMENT

Clinical score and biochemical indices-There was a significant relation between the clinical score, SHBG, FT3I, and FT4I when basal results and those during the first 12 weeks of treatment were? considered together (fig 3 ). Since the relations were generally not linear, correlation coefficients are not presented. In every case the FT3I and SHBG had returned to normal while the clinical score was 
still in the thyrotoxic range. The TRH response was suppressed despite clinical euthyroidism, and, conversely, it returned in three patients who remained clinically thyrotoxic.

Relation between biochemical indices-Since all indices returned to the normal range after starting carbimazole it is not surprising that significant correlations were seen between most of them during treatment. Again these were generally not linear. The TRH response was initially absent and remained so for a mean of 15 weeks (range 2-64). It is of interest to note the relation between the return of each index to normal and the return of pituitary responsiveness to TRH (fig 4). The TRH response was always absent until the FT4I had become normal. Similarly the FT3I was raised only on three occasions out of 55 when the TRH response had returned. There was likewise a close agreement between the fall in SHBG and the return of TRH responsiveness in both sexes. Subnormal SHBG concentrations were seen in association with the exaggerated TRH response on treatment only in the thyrotoxic men.

\section{OBSERVATIONS AFTER CARBIMAZOLE WAS DISCONTINUED}

Two patients became thyrotoxic again after treatment had stopped. In one the sequence seen on treatment was reversed, with early biochemical and delayed clinical evidence of thyrotoxicosis. FT3I and FT4I became raised first, followed one week later by a suppressed TRH response and raised SHBG. Clinical thyrotoxicosis was evident one week later. The other patient became thyrotoxic and was followed in another centre.

\section{Discussion}

Our findings indicate that thyroid hormone production, and therefore free T4 and T3 indices, fell rapidly on treatment with carbimazole. This was followed by a slightly slower fall in SHBG from raised levels. When all three biochemical values were normal the thyrotrophin response to TRH usually returned to normal, although later it became exaggerated in some patients. The clinical response was more gradual and the clinical state appeared to be less closely related to any of the biochemical indices studied than these were to one another.

On treatment none of the patients were clinically hypothyroid, although exaggerated thyrotrophin responses to TRH were sometimes seen. These were generally associated with low or low normal FT4I levels and variable (even occasionally raised) FT3I levels during the early phases of treatment. For a few weeks early in treatment these findings were seen despite continuing clinical thyrotoxicosis. Conversely, clinical euthyroidism was seen in two subjects despite biochemical evidence of continuing borderline hyperthyroidism.

Although a suppressed thyrotrophin response to TRH is a useful indication of thyrotoxicosis, the clinically euthyroid state may clearly be present for up to 47 weeks without a response to the releasing hormone. This agrees with the findings of others. ${ }^{1415}$

Our data show a high degree of internal consistency between the biochemical criteria and indicate that the main inconsistency during the early stages of medical treatment in thyrotoxicosis is between the clinical state and all the biochemical changes. As an aid to adjusting the doses of antithyroid drugs there is little to choose between measuring the thyroid hormones and measuring the indicators of tissue sensitivity - the pituitary thyrotrophin response to TRH and the hepatic production of SHBG. SHBG levels, however, have a graded response in patients with hyperthyroidism during treatment, whereas the thyrotrophin response is completely absent until thyroid hormone concentrations are normal and then rapidly overswings into the hypothyroid range.

Our data give further evidence that during the early treatment phase of thyrotoxicosis the clinical manifestations are not closely related to the degree of thyroid hormone excess at any particular point in time. In view of this discrepancy, however, the problem of determining the appropriate treatment of biochemical and clinical differences remains. We suggest that in such patients the dose of carbimazole that maintains the thyroid hormones and thyrotrophin concentrations within the normal range should be continued. If the clinical features indicate thyrotoxicosis (despite normal biochemical values) they should be treated independently with doses of a beta-adrenergic blocking drug large enough to remove the attendant signs and symptoms. The occasional patient with clinical thyrotoxicosis and biochemical findings suggestive of hypothyroidism may benefit from the introduction of beta-blocking drugs with a reduction in the dose of carbimazole to ensure a return of the biochemistry to normal. Patients with clinical euthyroidism but biochemical findings indicative of thyrotoxicosis might benefit from an earlier outpatient appointment so that the dose of carbimazole may be increased before clinical features reappear. The occurrence of clinical euthyroidism with circulating thyroid hormone and thyrotrophin concentrations usually associated with clinical hypothyroidism may require a reduction in the dose of carbimazole before such features result.

Clearly clinical assessment of the patient is an essential component in the management of thyrotoxicosis, and decisions based on biochemical or clinical features in isolation may lead to errors in treatment.

We are grateful to the staff of the metabolic ward, St Bartholomew's Hospital, for their co-operation. The studies were supported by the Medical Research Council, the Joint Research Board of St Bartholomew's Hospital and the Peel Medical Research Trust.

\section{References}

${ }^{1}$ Crooks, J, Wayne, E J, and Robb, R A, Lancet, 1960, 1, 397.

${ }^{2}$ Billewicz, W Z, et al, Quarterly fournal of Medicine, 1969, 38, 255.

3 Crépy, O, Dray, F, and Sebaoun, J, Comptes Rendus de l'Académie des Sciences, 1967, 264, 2651.

4 Gordon, G G, et al, Fournal of Clinical Endocrinology and Metabolism, 1969, 29, 164.

${ }^{5}$ Anderson, D C, Clinical Endocrinology, 1974, 3, 69.

${ }^{6}$ Ruder, H, et al, Fournal of Clinical Endocrinology and Metabolism, 1971, 33, 382 .

'Southren, A L, et al, fournal of Clinical Endocrinology and Metabolism, 1974, 38, 207.

${ }^{8}$ Rosner, W, fournal of Clinical Endocrinology and Metabolism, 1972, 34, 983.

${ }^{9}$ Simpson, D, Clinical Chemistry, 1967, 13, 890.

10 Clark, F, and Horn, D B, Fournal of Clinical Endocrinology and Metabolism, $1965,25,39$.

${ }^{11}$ Chan, V, et al, Annals of Clinical Biochemistry, 1975, 12, 173.

${ }_{12}$ Mortimer, C H, et al, Clinical Endocrinology, 1974, 3, 97.

13 Ormston, B J, et al, Lancet, 1971, 2, 10.

14 Von Zur Mühlen, A, et al, Acta Endocrinologica (Kфbenhavn), 1971, 155, suppl p 6.

${ }^{15}$ Hesch, R D, et al, Acta Endocrinologica (Kфbenhavn), 1974, 75, 514.

(Accepted 18 November 1976) 